\title{
Enhanced ABSF Algorithm with a Relay Function in LTE Heterogeneous Networks
}

\author{
Lehung Nguyen ${ }^{1}\left(\mathbb{D}\right.$ and Sungoh Kwon ${ }^{2, *(1)}$ \\ 1 BNF Technology Inc., Daejeon 34028, Korea; lehung.nguyen.27@gmail.com \\ 2 School of Electrical Engineering, University of Ulsan, Ulsan 44610, Korea \\ * Correspondence: sungoh@ulsan.ac.kr
}

Received: 10 July 2020; Accepted: 16 August 2020; Published: 19 August 2020

\begin{abstract}
In this study, we enhance the almost blank subframe (ABSF) algorithm in a Long Term Evolution (LTE) heterogeneous network (HetNet) by providing a relay function. The ABSF is a technique proposed by the Third Generation Partnership Project to reduce interference in a HetNet. Despite the fact that the ABSF effectively mitigates intercell interference, it has two major disadvantages. First, the ABSF algorithm alters the scheduling policy of macro base stations. Second, it degrades the capacity of users served by femto base stations. Our proposed enhanced algorithm applies a relay function to assist victim macro user equipment (VMUE), and it reduces the side effects caused by the ABSF algorithm. Taking resource allocation and power control into account, the relay function assists VMUE in such a way that interference with other users is minimized. Via simulation results, the proposed algorithm exhibits improvements of $18 \%$ and $8 \%$ for system throughput and user satisfaction, respectively, in comparison with the conventional ABSF.
\end{abstract}

Keywords: LTE; heterogeneous network; intercell interference; ABSF; relay function; resource allocation; power control

\section{Introduction}

Recent years have seen a dramatic growth in mobile data traffic. A Cisco Systems Inc. forecast suggests that the number of activated mobile devices will reach 11.6 billion by 2021 [1]. At that time, mobile data traffic will be 49 exabytes per month, which is seven times that seen in 2016 . The increasing data traffic demand motivates the development of the next generation of cellular mobile networks with many advanced features. Among these improvements, two features that draw significant concern in the literature are the heterogeneous network (HetNet) and relay functionality [2].

In a HetNet, in addition to large macro base stations (macrocells), low-power base stations (small cells) have been introduced as a solution to improve network performance by offloading traffic from the macro base stations [3]. More specifically, the term small cells is used to refer to pico and femto base stations in Long Term Evolution (LTE) HetNets [4]. Pico base stations are designed for outdoor use, and cover a range of several hundred meters [5]. They are deployed in areas with heavy mobile data traffic, and are open to public users (open-subscriber groups). Conversely, since femto base stations are utilized for private use, they have a smaller coverage area and only serve registered users [6]. In addition, taking advantage of a relay function, user equipment (UE) retransmit data of other UE in close proximity, contributing to offloading mobile data from macro base stations, as well as reducing the transmit power needed. Despite the fact that a HetNet provides many advantages, it also comes with disadvantages, such as handover management [7,8], load balancing [9], and especially intercell interference.

Aiming to maximize spectral efficiency, macrocells and small cells transmit in the same resource block but interfere with each other. There are several situations wherein the interference may arise, 
including the co-tier interference and cross-tier interference [10]. The co-tier interference addresses the interference between network elements on the same tier. For instance, the interference between adjacent macrocells or the interference between neighboring small cells, attempting to use the same resource simultaneously, are considered as co-tier interference. The cross-tier interference sees interference between network elements belonging to different tiers like macrocells and small cells under two different scenarios. In the first one, a strong signal from macrocells interferes with UE that are served by pico base stations [11]. In the second, femto base stations operating in closed-subscriber group (CSG) mode prevent nearby macro UE from associating with either femto or macro base stations [12]. Since these macro users are not members of the CSG, they are not served by the femto base stations. Moreover, their desired signals are interrupted by signals from the femto base stations. Figure 1 illustrates two scenarios of intercell interference between macrocells and small cells. Unlike the conventional single-tier cellular networks, the interference issue in the cross-tier HetNets is significantly challenging [3]. The various types of base stations require different bandwidths and delay constraints of the backhaul network. For example, since femto base stations are unlikely to be connected directly to the core network, limited backhaul signaling is available for interference coordination. Furthermore, the limited access control of small cells may prevent users from handing over to the nearest cell, leading to strong interference scenarios in both uplink and downlink. In addition, continuous sensing and monitoring of the radio environment to adapt and mitigate interference may result in high energy consumption.

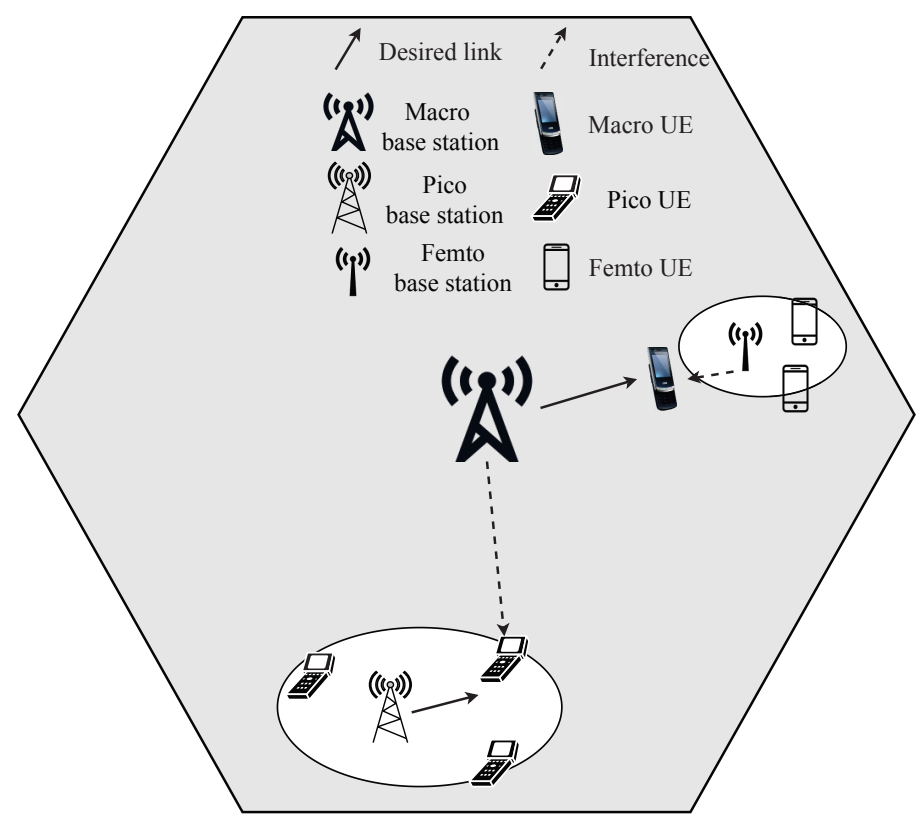

Figure 1. Two scenarios of intercell interference in HetNet.

Many solutions have been proposed to resolve the problem of intercell interference in the frequency and time domains. In the frequency domain, co-tier interference is avoided by allocating frequencies to multiple neighboring cells in such a way that the allocated spectrums do not overlap. This approach is referred to as the intercell interference coordination (ICIC) technique, which includes hard, soft, and fractional frequency schemes [13]. In the time domain, one of the effective solutions to deal with interference between macrocells and small cells is the almost blank subframe (ABSF), in which the aggressor and victim base stations share the same frequency spectrum but schedule their transmissions to avoid the conflicts [14,15]. Although ICIC schemes can effectively coordinate the interference between adjacent macrocells, spectrum efficiency decreases because of the increasing number of in-use frequency bands $[10,16]$. ABSF, conversely, fully optimizes spectral efficiency, but two major existing drawbacks have not been fully resolved. First, muting 
some of the femto base station's subframes over a period of time degrades the performance of UE associated with the femto base station [14]. Second, the scheduling policy of a victim macro base station has to be changed to correspond with the muting period of the femto base stations in order to serve the victim UE. Several studies have been conducted to improve the original ABSF. Some authors have aimed to find the optimal number of blank subframes in order to restrict the capacity degradation $[17,18]$. Furthermore, the reduced-power subframe was investigated as an alternative to the blank subframe [19]. In Reference [20], the authors proposed an approach to determine the adaptive amount of reduced power for each subframe. Although the quantity of blank or reduced-power subframes, and the amount of reduced power per subframe, were determined in previous work, the rich diversity in HetNets has not been fully utilized.

In a HetNet, there exist multiple-layer networks aiming to guarantee the best quality of service for users. Depending on the real-time status of the network, macro users are served by macro base stations or by a relay function. Thanks to high density and the closer proximity of mobile users, a relay function is capable of providing better and more reliable service to users when the macro base stations are overloaded or interrupted, thus helping to enhance network performance. In Reference [21], the authors provided a good survey of relay function in LTE HetNets. In Reference [22], the relay function is studied to enhance the quality of experience of LTE HetNets. The application of relay function in offloading traffic and mitigating congestion for HetNets is addressed in Reference [23]. In addition, the relay function was considered as part of an interference forwarding scheme in a dual link for enhancing the signal-to-interference-plus-noise-ratio (SINR) at victim UE in HetNets [24]. In Reference [25], the authors provided an analysis of joint relay selection, full-duplex, and relay function in non orthogonal multiple access networks. Nonetheless, ABSF was not taken into account in these works. As an important feature introduced in LTE advanced networks, a relay function also has a great potential to significantly improve the communication experiences, especially in the ultra dense HetNets with an extremely large number of small cells. However, available works exploring the relay-function-based applications jointly with ABSF were limited in literature, and therefore, more research efforts are encouraged towards this topic.

The main contribution of this paper is to fully exploit the use of a relay function to improve the weak points of the ABSF that were not covered in conventional approaches. In other words, ABSF and the relay function are considered in a joint manner within the HetNet environment to assist victim UE. This research also considers the optimal resource allocation strategy for the relay function in order to minimize interference with other users. The performance of the algorithm is then demonstrated via simulation results.

The rest of this paper is organized as follows. Section 2 describes the system model. Section 3 formulates the problem caused by ABSF. In Section 4, we propose the algorithm to improve the above-mentioned drawbacks by taking advantage of a relay function. Section 5 compares the performance of the proposed algorithm with existing approaches. Section 6 concludes the paper.

\section{System Model}

\subsection{LTE Heterogeneous Networks}

We consider a HetNet with macro base stations $M_{k} \mathrm{~s}, k \in\left\{1, \ldots N_{M}\right\}$, and femto base stations $F_{j}^{k} \mathrm{~s}$, $j \in\left\{1, \ldots N_{F}\right\} . F_{j}^{k}$ s are located within the macro base station $M_{k}$ coverage area and share the same common transmission bandwidth to maximize spectral efficiency [26]. Macro UE $m_{i}^{k} \mathrm{~s}, i \in\left\{1, \ldots N_{m}\right\}$, and femto $\mathrm{UE} f_{n}^{(k, j)}$ s, $n \in\left\{1, \ldots N_{f}\right\}$, are served by macro base station $M_{k}$ and femto base station $F_{j}^{k}$, respectively. $N_{M}, N_{F}, N_{m}$, and $N_{f}$ represent the quantity of macro base stations, femto base stations, macro UE, and femto UE, respectively. Each macro UE can play the role of relay node to forward data to other macro UE. The femto base stations $F_{j}^{k}$ s operate in CSG mode and serve only the registered femto UE $f_{n}^{(k, j)}$ s. Nearby are macro UE, but they are not registered members of CSG, and are not served by the femto base stations. 
A macro base station measures the SINR values of all macro UE to detect victim macro user equipment (VMUE). The SINR of macro UE $m_{i}^{k}$, served by macro base station $M_{k}$ and suffering interference from femto base stations $F_{j}^{k} \mathrm{~s}$, is given by

$$
\gamma_{m_{i}^{k}}^{M_{k}}(0)=\frac{P_{M_{k}} G_{\left(M_{k}, m_{i}^{k}\right)}}{I_{M_{\left(m_{i}^{k}\right)}}+I_{F_{\left(m_{i}^{k}\right)}}(0)+\sigma_{m_{i}^{k}}},
$$

where $\sigma_{m_{i}^{k}}, I_{M_{\left(m_{i}^{k}\right)}}$, and $I_{F_{\left(m_{i}^{k}\right)}}(0)$ denote the thermal noise, interference from adjacent macro base stations $M_{k^{\prime}} \mathrm{s}\left(k^{\prime} \neq k\right)$, and interference from femto base stations $F_{j}^{k} \mathrm{~s}$ with macro UE $m_{i}^{k}$, respectively. Interference $I_{M_{\left(m_{i}^{k}\right)}}$ and $I_{F_{\left(m_{i}^{k}\right)}}(0)$ are expressed as

$$
I_{M_{\left(m_{i}^{k}\right)}}=\sum_{M_{k^{\prime}} \in \mathcal{M}, k^{\prime} \neq k} P_{M_{k^{\prime}}} G_{\left(M_{k^{\prime}}, m_{i}^{k}\right)^{\prime}}
$$

and

$$
I_{F_{\left(m_{i}^{k}\right)}}(0)=\sum_{F_{j}^{k}} P_{F_{j}^{k}} G_{\left(F_{j}^{k}, m_{i}^{k}\right)}
$$

where $P_{M_{k^{\prime}}}$ and $P_{F_{j}^{k}}$ represent the transmit power of macro base station $M_{k^{\prime}}$ and femto base station $F_{j}^{k}$. Two parameters $G_{\left(M_{k^{\prime}}, m_{i}^{k}\right)}$ and $G_{\left(F_{j}^{k}, m_{i}^{k}\right)}$, denote the channel gain between macro base station $M_{k^{\prime}}$ and VMUE $m_{i}^{k}$, and between femto base station $F_{j}^{k}$ and VMUE $m_{i}^{k}$, respectively. The channel gain between base station and UE is calculated based on distant-dependent macroscopic pathloss, shadow fading pathloss (location-dependent), and the antenna gain of each base station. Similarly, the SINR of femto $\mathrm{UE} f_{n}^{(k, j)}$ served by $F_{j}^{k}$ is

$$
\gamma_{f_{n}^{(k, j)}}(0)=\frac{P_{F_{j}^{k}} G_{\left(F^{k}, f_{n}^{(k, j)}\right)}}{I_{M_{\left(f_{n}^{(k, j)}\right)}}+I_{F_{\left(f_{n}^{(k, j)}\right)}}(0)+\sigma_{f_{n}^{(k, j)}}},
$$

where $I_{\left.M_{\left(f_{n}(k, j)\right.}\right)}$ and $I_{F_{\left(f_{n}^{(k, j)}\right)}}(0)$ express the interference from macro base stations $M_{k} \mathrm{~s}$ and femto base stations $F_{j^{\prime}}^{k}\left(j^{\prime} \neq j\right)$ with the femto $\operatorname{UE} f_{n}^{(k, j)}$. Since each femto base station only occupies a narrow spectrum to serve a limited number of users, the interference between femto base stations is mostly

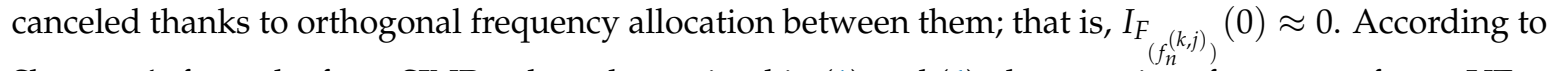
Shannon's formula, from SINR values determined in (1) and (4), the capacity of macro or femto UE $u$, $u \in\left\{m_{i}^{k}, f_{n}^{(k, j)}\right\}$ is

$$
C_{u}=\log _{2}\left(1+\gamma_{u}\right)
$$

In a HetNet, the femto base stations, that interfere with macro users, are referred as femto aggressors. High interference from femto aggressors significantly reduces $\gamma_{m_{i}^{k}}^{M_{k}}(0)$. If the SINR value of macro UE $m_{i}^{k}$ is less than a certain threshold, i.e., $\gamma_{m_{i}^{k}}^{M^{k}}(0) \leq \gamma_{\text {thres }}$, it is considered a VMUE [27], and is denoted as $m_{(i, v)}^{k}$. The SINR of VMUE $m_{(i, v)}^{k}$ served by macro base station $M_{k}$ is given by

$$
\gamma_{m_{(i, v)^{k}}^{M_{k}}}(0)=\frac{P_{M_{k}} G_{\left(M_{k}, m_{(i, v)}^{k}\right)}}{I_{M_{\left(m_{(i, v)}^{k}\right)}}+I_{F_{\left(m_{(i, v)}^{k}\right)}}(0)+\sigma_{m_{(i, v)}^{k}}}
$$




\subsection{ABSF in a Clustered Network}

For satisfactory performance of VMUE, a macro base station enables ABSF to mitigate interference. To cut off interfering transmission time, ABSF mutes a fraction of the aggressors' subframes, and hence, decreases the cumulative interfering power. The benefits of ABSF is undeniable; however, muting some subframes of femto base stations degrades their capacity to serve femto UE. Consequently, determining the appropriate ABSF ratio is critical. Previous work $[17,18]$ derived the optimal ABSF ratio based on the number of users, including macro users and femto users, but these approaches may not guarantee performance for victim macro users. In this paper, we derive the optimal ABSF ratio, $\alpha_{m_{(i, v)}^{k}}$, to satisfy each victim macro user, $m_{(i, v)}^{k}$, as proposed in [27]. In a dense HetNet, the number of femto base stations is extremely large and a single VMUE may suffer interference from multiple femto base stations. Therefore, in order to effectively reduce interference, femto base stations with mutual VMUE are grouped into the same cluster to be synchronously muted during the proper subframes. ABSF ratio $\alpha_{C_{l}^{k}}$ for each cluster, $C_{l}^{k}$, of femto base stations is determined based on the $\alpha_{m_{(i, v)}^{k}}$ s ABSF ratios of all VMUE belonging to victim list $\mathcal{V}_{l}^{k}$ of the cluster

$$
\alpha_{C_{l}^{k}}=\max \left\{\alpha_{m_{(i, v)}^{k}}\right\}, \forall m_{(i, v)}^{k} \in \mathcal{V}_{l}^{k}
$$

$\mathrm{ABSF}$ ratio $\alpha_{F_{j}^{k}}$ represents the muted power fraction of femto aggressors $F_{j}^{k} \mathrm{~s}\left(0 \leq \alpha_{F_{j}^{k}} \leq 1\right)$, and is expressed as

$$
\alpha_{F_{j}^{k}}= \begin{cases}0, & \text { No ABSF } \\ 0<\alpha_{F_{j}^{k}} \leq 1, & \text { ABSF enabled }\end{cases}
$$

Since a femto base station may be a member of multiple clusters, ABSF ratio $\alpha_{F_{j}^{k}}$ of femto base station $F_{j}^{k}$ is derived based on the ABSF ratios $\alpha_{C_{l}^{k}}$ s of all clusters $C_{l}^{k}$ s to which femto base station $F_{j}^{k}$ belongs

$$
\alpha_{F_{j}^{k}}=\max \left\{\alpha_{C_{l}^{k}}\right\}, \forall C_{l}^{k} \ni F_{j}^{k}
$$

In other words, $\mathrm{ABSF}$ ratio $\alpha_{F_{j}^{k}}$ for each femto aggressor $F_{j}^{k}$ is determined based on the demanded ratio of all VMUE $m_{(i, v)}^{k} s$ belonging to victim list $\mathcal{V}_{j}^{k}$ interfered with by $F_{j}^{k}$ as

$$
\alpha_{F_{j}^{k}}=\max \left(\alpha_{m_{(i, v)}^{k}}\right), \forall m_{(i, v)}^{k} \in \mathcal{V}_{j}^{k} .
$$

After activating the ABSF with ratio $\alpha_{F_{j}^{k}}$ for femto base station $F_{j}^{k}$, the transmission-activated time and cumulative transmit power of $F_{j}^{k}$ are decreased by $\alpha_{F_{j}^{k}}$ ratio [27]. Therefore, after activating the ABSF, the SINR of VMUE $m_{(i, v)}^{k}$ served by macro base station $M_{k}$ is given by

$$
\gamma_{m_{(i, v)}^{k}}^{M_{k}}\left(\alpha_{F_{j}^{k}}\right)=\frac{P_{M_{k}} G_{\left(M_{k}, m_{(i, v)}^{k}\right)}}{I_{M_{\left(m_{(i, v)}^{k}\right)}+}+I_{F_{\left(m_{(i, v)}^{k}\right)}}\left(\alpha_{F_{j}^{k}}\right)+\sigma_{m_{(i, v)}^{k}}},
$$

where

$$
I_{F_{\left(m_{(i, v)}^{k}\right)}}\left(\alpha_{F_{j}^{k}}\right)=\sum_{F_{j}^{k}} G_{\left(F_{j}^{k}, m_{(i, v)}^{k}\right)} P_{F_{j}^{k}}\left(1-\alpha_{F_{j}^{k}}\right) .
$$


Similarly, after activating the ABSF, the SINR of femto $\operatorname{UE} f_{n}^{(k, j)}$ served by femto base station $F_{j}^{k}$ is

$$
\begin{aligned}
& \gamma_{f_{n}^{(k, j)}}\left(\alpha_{F_{j}^{k}}\right)= \frac{P_{F_{j}^{k}}\left(1-\alpha_{\left.F_{j}^{k}\right)}\right) G_{\left(F_{j}^{k}, f_{n}^{(k, j)}\right)}}{I_{M\left(f_{n}^{(k, j)}\right)}{ }^{+I_{F}}\left(f_{n}^{(k, j)}\right)\left(\alpha_{F_{j}^{k}}\right)+\sigma_{f_{n}^{(k, j)}}} \\
&=\gamma_{f_{n}^{(k, j)}}(0)\left(1-\alpha_{F_{j}^{k}}\right) .
\end{aligned}
$$

\subsection{Relay Function}

In addition to a macro base station, a macro UE can be served by another macro UE using a relay function. Thanks to the closer proximity between a relay node and the served node, a higher SINR value can be achieved with lower energy consumption. Therefore, a relay function, in full-duplex or half-duplex mode, is a promising way to assist critical UE such as victim macro users. To assist VMUE $m_{(i, v)}^{k}$, a macro base station selects one UE among the other macro UE, to play the role of relay node, denoted as $m_{(i, v, r)}^{k}$. The SINR value of VMUE $m_{(i, v)}^{k}$ served by relay node $m_{(i, v, r)}^{k}$ is expressed as

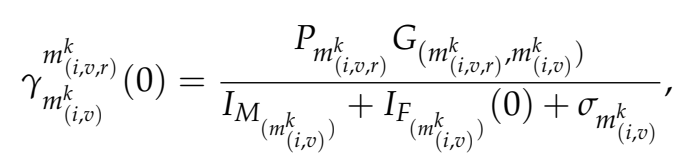

where $P_{m_{(i, v, r)}^{k}}$ denotes the transmit power of relay node $m_{(i, v, r)}^{k}$, and $G_{\left(m_{(i, v, r)}^{k}, m_{(i, v)}^{k}\right)}$ denotes the channel gain between relay node $m_{(i, v, r)}^{k}$ and VMUE $m_{(i, v)}^{k}$. From (11) and (14), the SINR of victim macro UE $m_{(i, v)}^{k}$ is expressed as

$$
\gamma_{m_{(i, v)}^{k}}(0)=\left\{\begin{array}{l}
\gamma_{m_{(i, v)}}^{M_{k}}(0), \text { if } m_{(i, v)}^{k} \text { is served by } M_{k} \\
\gamma_{(i, v, r)}^{k}(0), \text { if } m_{(i, v)}^{k} \text { is served by } m_{(i, v, r)}^{k}
\end{array}\right.
$$

However, assisting VMUE with relays also causes interference with macro UE that share their resource blocks with relay nodes. In particular, we denote $m_{(i, v, r, s)}^{k}$ as the macro UE that shares a resource block with relay node $m_{(i, v, r)}^{k}$. In downlink multi-user orthogonal frequency-division multiplexing LTE systems, each subcarrier should only be assigned to only one user who has the best channel gain for that subcarrier [28]. Therefore, each relay node $m_{(i, v, r)}^{k}$, that occupies one subcarrier, only interferes with one macro UE. The SINR value of affected macro UE $m_{(i, v, r, s)}^{k}$ is calculated as

$$
\frac{\gamma_{m_{(i, v, r, s)}^{k}}(0)=}{P_{M_{k} G_{\left(M_{k}, m_{(i, v, r, s}^{k}\right)}^{k}}} \frac{}{I_{R}\left(m_{(i, v, r, s)}^{k}\right)+I_{M}\left(m_{(i, v, r)}^{k}\right)+I_{F}\left(m_{(i, v, r, s)}^{k}\right)(0)+\sigma_{m_{(i, v, r, s)}^{k}}^{k}},
$$

where $I_{R}\left(m_{(i, v, r, s)}^{k}\right)$ denotes the interference from relay node $m_{(i, v, r)}^{k}$ that affects macro UE $m_{(i, v, r, s)}^{k}$ and it is expressed as

$$
I_{R}\left(m_{(i, v, r, s)}^{k}\right)=P_{m_{(i, v, r)}^{k}} G_{\left(m_{(i, v, r)}^{k}, m_{(i, v, r, s)}^{k}\right)} .
$$

A summary of all notations used in this paper is in Table 1. 
Table 1. Summary of notations.

\begin{tabular}{cl}
\hline Notation & Meaning \\
\hline$M_{k}$ & Macro base station \\
$N_{M}$ & Number of macro base stations \\
$m_{i}^{k}$ & Macro UE $i$ served by macro base station $M_{k}$ \\
$m_{(i, v)}^{k}$ & Macro UE $i$ marked as victim macro UE $v$ \\
$m_{(i, v, r)}^{k}$ & Macro UE chosen as relay node $r$ for victim macro UE $v$ \\
$m_{(i, v, r, s)}^{k}$ & Affected macro UE $s$ sharing resources with relay node $r$ \\
$N_{m}^{k}$ & Number of macro UE \\
$N_{m}^{k}$ & Number of macro UE served by macro base station $M_{k}$ \\
$N_{v}^{k}$ & Number of macro victim UE served by macro base station $M_{k}$ \\
$F_{j}^{k}$ & Femto base station $j$ within the coverage area of macrocell $M_{k}$ \\
$N_{F}$ & Number of femto base stations \\
$\mathcal{V}_{j}^{k}$ & Set of victim UE interfered with by $F_{j}^{k}$ \\
$f_{n}^{(k, j)}$ & Femto UE $n$ served by femto base station $F_{j}^{k}$ \\
$N_{f}$ & Number of femto UE \\
\hline
\end{tabular}

\section{Problem Formulation}

The ABSF notably improves the signal quality of VMUE. However, it has two major drawbacks. First, on the macro base station side, despite the fact that it is not necessary to introduce an ABSF, macro base station $M_{k}$ has to change its scheduling policy to serve VMUE on the subframes that are left blank by femto aggressors. Changing the scheduling policy leaves negative consequences for the performance of macro base station $M_{k}$. In an empirical network, the large amount of VMUE and femto aggressors exerts a significant influence on the scheduling policy of macro base stations. Secondly, on the femto base station side, although ABSF ratio $\alpha_{F_{j}^{k}}$ has been selected carefully, this technique somewhat decreases the transmit power of femto base stations $F_{j}^{k} \mathrm{~s}$, which results in degradation in the performance of femto UE $f_{n}^{(k, j)}$ s.

Our objective is to minimize the resource loss of femto UE due to ABSF, while still satisfying the SINR condition of VMUE $m_{(i, v)}^{k}$ s, i.e., $\gamma_{m_{(i, v)}^{k}}(0) \geq \gamma_{\text {thres }}$ [29]. From (15), the VMUE $m_{(i, v)}^{k}$ can be served by macro base station $M_{k}$ or relay node $m_{(i, v, r)}^{k}$, hence, the problem can be formulated as

$$
\begin{array}{ll}
\min _{\alpha_{F_{j}^{k}}} & \sum_{F_{j}^{k}} \sum_{f_{n}^{(k, j)}} \log _{2}\left(\frac{1+\gamma_{f_{n}^{(k, j)}}(0)}{1+\gamma_{f_{n}^{(k, j)}}(0)\left(1-\alpha_{F_{j}^{k}}\right)}\right) \\
\text { s.t. } & \gamma_{m_{k(i, v)}^{k}}^{M_{k}}(0) \geq \gamma_{\text {thres }}, \text { if } m_{(i, v)}^{k} \text { is served by } M_{k}, \\
& \gamma_{m_{(i, v)}^{k}}^{k}(0) \geq \gamma_{\text {thres }}, \text { if } m_{(i, v)}^{k} \text { is served by } m_{(i, v, r)}^{k}, \\
& 0 \leq \alpha_{m_{(i, v)}^{k}}^{k} \leq 1, \forall m_{(i, v)^{\prime}}^{k}, \\
& 0 \leq \alpha_{F_{j}^{k}} \leq 1, \forall F_{j}^{k} .
\end{array}
$$

The resource loss of femto users in (18) is estimated and modified to reduce its dependency. The manipulations are performed step by step as follows. First, we attempt to estimate the capacity degradation due to the ABSF. According to (4), (5), and (13), the capacity degradation of a single femto $\mathrm{UE} f_{n}^{(k, j)}$ served by femto base station $F_{j}^{k}$ before and after applying ABSF is

$$
\begin{aligned}
\Delta_{f_{n}^{(k, j)}} & =C_{f_{n}^{(k, j)}}(0)-C_{f_{n}^{(k, j)}}\left(\alpha_{F_{j}^{k}}\right) \\
& =\log _{2}\left(\frac{1+\gamma_{f_{n}^{(k, j)}}(0)}{1+\gamma_{f_{n}^{(k, j)}}(0)\left(1-\alpha_{F_{j}^{k}}\right)}\right),
\end{aligned}
$$


where $C_{f_{n}^{(k, j)}}(0)$ and $C_{f_{n}^{(k, j)}}\left(\alpha_{F_{j}^{k}}\right)$ represent the capacity of a femto UE before and after the ABSF, respectively. The capacity degradation of all femto UE is

$$
\Delta_{C}^{\mathrm{Total}}=\sum_{F_{j}^{k}} \sum_{f_{n}^{(k, j)}} \log _{2}\left(\frac{1+\gamma_{f_{n}^{(k, j)}}(0)}{1+\gamma_{f_{n}^{(k, j)}}(0)\left(1-\alpha_{F_{j}^{k}}\right)}\right) .
$$

We define the function $g\left(\alpha_{F_{j}^{k}}\right)$ as

$$
g\left(\alpha_{F_{j}^{k}}\right)=\log _{2}\left(\frac{1+\gamma_{f_{n}^{(k, j)}}(0)}{1+\gamma_{f_{n}^{(k, j)}}(0)\left(1-\alpha_{F_{j}^{k}}\right)}\right) .
$$

The second derivative of $g\left(\alpha_{F_{j}^{k}}\right)$ is

$$
g^{\prime \prime}\left(\alpha_{F_{j}^{k}}\right)=\frac{\gamma_{f_{n}^{(k, j)}}(0)}{1+\gamma_{f_{n}^{(k, j)}}(0)\left(1-\alpha_{F_{j}^{k}}\right)} .
$$

Since $\gamma_{f_{n}^{(k, j)}}(0)>0, \forall f_{n}^{(k, j)}$ and $0 \leq \alpha_{F_{j}^{k}} \leq 1, \forall F_{j}^{k}, g^{\prime \prime}\left(\alpha_{F_{j}^{k}}\right)$ is positive. Hence, $g\left(\alpha_{F_{j}^{k}}\right)$ is convex with respect to $\alpha_{F_{j}^{k}}$, so the local minimum is also the global minimum. In the interval $[0,1]$, as $\alpha_{F_{j}^{k}}$ increases, the value of $\Delta_{C}^{\text {Total }}$ decreases. Therefore, we minimize the ABSF ratio applied on all femto aggressors $\alpha_{F_{j}^{k}} \mathrm{~s}$.

The capacity degradation of femto UE $f_{n}^{(k, j)}$ s served by femto base stations $F_{j}^{k}$ s is lowered by reducing the required ABSF ratios $\alpha_{F_{j}^{k}} \mathrm{~S}$ of these femto base stations. From (10), these values are determined by the maximum ABSF ratio required by critical VMUE, which suffers from the strongest interference $\max \left(\alpha_{m_{(i, v)}^{k}}\right)$.

To reduce the maximum required ABSF ratio, we consider a relay function to assist these critical VMUE if a relay can provide better signal quality than a macro base station, consequently, giving rise to lower capacity degradation. However, the efficiency of the relay function significantly depends on the relay selection, the channel gain between the relay node and VMUE, and relay transmit power while keeping the interference from the relay lower than the tolerance of the affected macro UE. Optimization problem (18) can be restated as

$$
\begin{array}{ll}
\min _{\gamma_{(i, v)}^{k}} & \max \left(\alpha_{m_{(i, v)}^{k}}\right), \forall m_{(i, v)}^{k} \\
\text { s.t. } & \gamma_{m_{(i, v)}^{k}}^{M_{k}}(0) \geq \gamma_{\text {thres }}, \text { if } m_{(i, v)}^{k} \text { is served by } M_{k \prime}, \\
& \gamma_{m_{(i, v)}^{k}}^{m_{(i, v)}^{k}}(0) \geq \gamma_{\text {thres, }} \text { if } m_{(i, v)}^{k} \text { is served by } m_{(i, v, r) \prime}^{k}, \\
& \gamma_{m_{(i, v, r, s)}^{k}}^{k}(0) \geq \gamma_{\text {thres }}, \text { if } m_{(i, v)}^{k} \text { is served by } m_{(i, v, r)}^{k}, \\
& 0 \leq \alpha_{m_{(i, v)}^{k}} \leq 1, \forall m_{(i, v)^{\prime}}^{k} \\
& 0 \leq \alpha_{F_{j}^{k}} \leq 1, \forall F_{j}^{k} .
\end{array}
$$

\section{Proposed Algorithm}

This section explains the algorithm to minimize the maximum required ABSF ratio max $\left(\alpha_{m_{(i, v)}^{k}}\right)$, $\forall m_{(i, v)}^{k}$ of all victim macro users of macro base station $M_{k}$. The overall algorithm is given in Algorithm 1. 
The proposed algorithm performs based on these following steps. First, macro base stations collect the SINR values of all served macro UE, and then detect which macro UE are VMUE as in Algorithm 2. All VMUE report their femto aggressors to their serving macro base station to complete the femto aggressors list. Macro base stations clarify the victim list of each femto aggressor and then group femto aggressors that have mutual VMUE into the same cluster. Secondly, for each cluster of femto aggressors, the macro base stations sort all the VMUE in the victim list in increasing order with respect to SINR, and decides which VMUE have the higher priority to receive the assistance of a relay function. Thirdly, macro base stations select the most appropriate relay node for each chosen VMUE in each cluster. The relay selection procedure is described in Algorithm 3. After that, the radio resource is allocated for relay nodes in such a way that the interference caused by relays is at a minimum. In the next step, the transmit power of relay nodes is adjusted to guarantee that the performance of affected macro UE, which share their resource blocks to relay function, is acceptable. This step is explained inl Algorithm 4. Lastly, after estimating the new SINR values of the VMUE served by relays, a macro base station decides whether the relay function should be enabled for this VMUE or not. Finally, the ABSF ratio is determined based on the new status of the VMUE. The whole procedure is executed in each transmission time interval. The details of each step are explained in the following sub-sections.

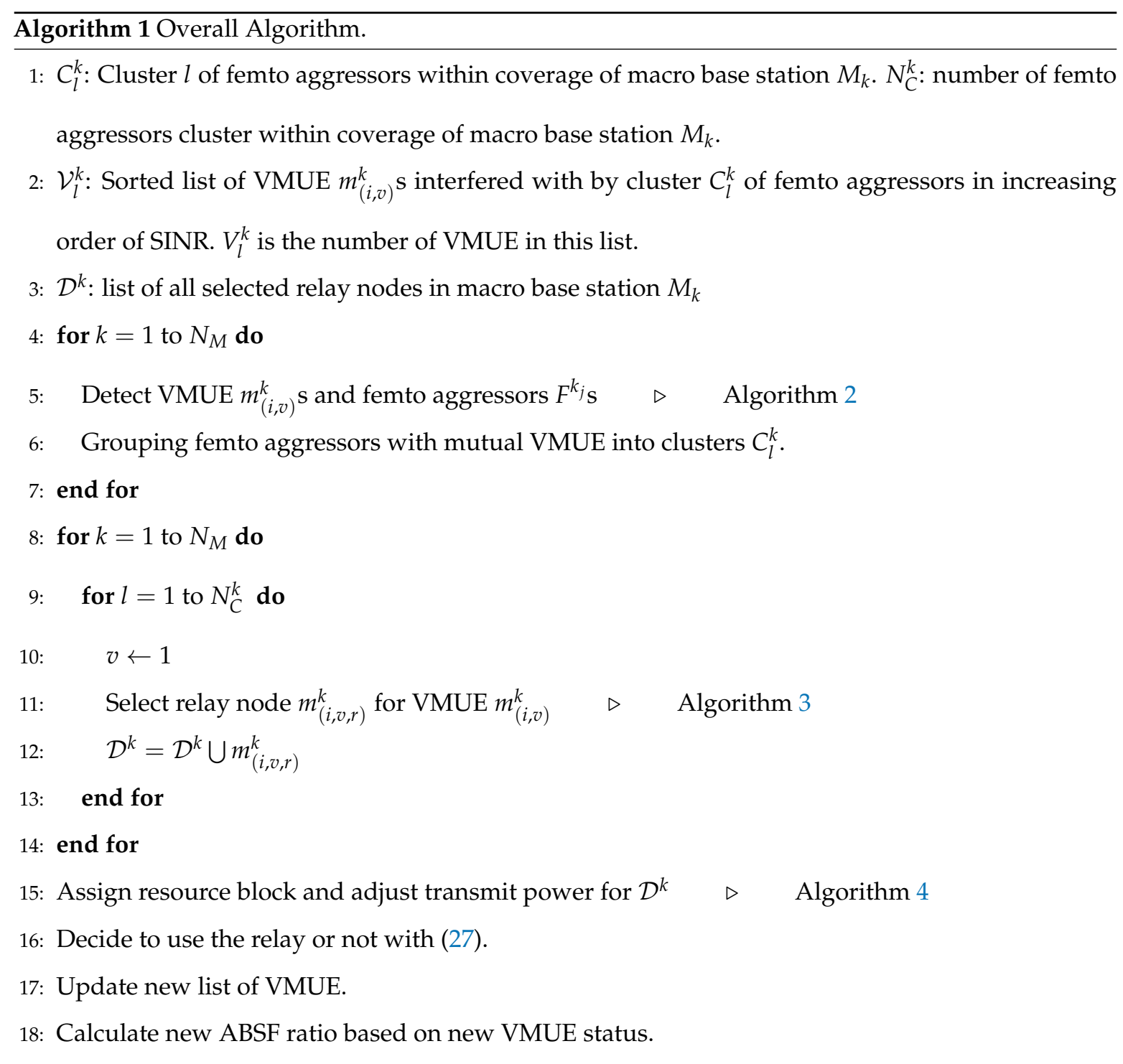




\subsection{Grouping Femto Aggressors}

Macro base stations detect VMUE by collecting the SINR values of all macro UE. Each VMUE sends information about its femto aggressors to its serving macro base station. The procedure for collecting VMUE and detecting femto aggressors is given in Algorithm 2. Based on that information, macro base stations group femto aggressors into clusters. More specifically, femto aggressors, that have the victim lists consisting of mutual VMUE, are grouped into a cluster. A femto aggressor may be a member of a single cluster or multiple clusters. For instance, in Figure 2, femto aggressor $F_{6}^{1}$ interferes VMUE $m_{(8,6)}^{1}$ and is considered a cluster. Two femto aggressors $F_{4}^{1}$ and $F_{5}^{1}$ interfere with two VMUE $m_{(6,5)}^{1}$ and $m_{(5,4)}^{1}$, and are grouped into a cluster. For three femto aggressors $F_{1}^{1}, F_{2}^{1}$, and $F_{3}^{1}$, their victim lists are $\mathcal{V}_{1}^{1}=\left\{m_{(1,1)}^{1}\right\}, \mathcal{V}_{2}^{1}=\left\{m_{(1,1)}^{1}, m_{(2,2)}^{1}\right\}$, and $\mathcal{V}_{3}^{1}=\left\{m_{(2,2)}^{1}, m_{(3,3)}^{1}\right\}$, respectively. Therefore, $F_{1}^{1}$ and $F_{2}^{1}$ are formed into a cluster with respect to VMUE $m_{1,1}^{1}$. Femto aggressors $F_{2}^{1}$, and $F_{3}^{1}$ are grouped into one cluster with respect to VMUE $m_{2,2}^{1}$. Femto aggressor $F_{3}^{1}$ forms a separate cluster with respect to VMUE $m_{(3,3)}^{1}$. In this case, there are five clusters in total, including $C_{1}^{1}=\left\{F_{1}^{1}, F_{1}^{2}\right\}, C_{2}^{1}=\left\{F_{1}^{2}, F_{1}^{3}\right\}$, $C_{3}^{1}=\left\{F_{1}^{3}\right\}, C_{4}^{1}=\left\{F_{1}^{4}, F_{1}^{5}\right\}$, and $C_{5}^{1}=\left\{F_{1}^{6}\right\}$.

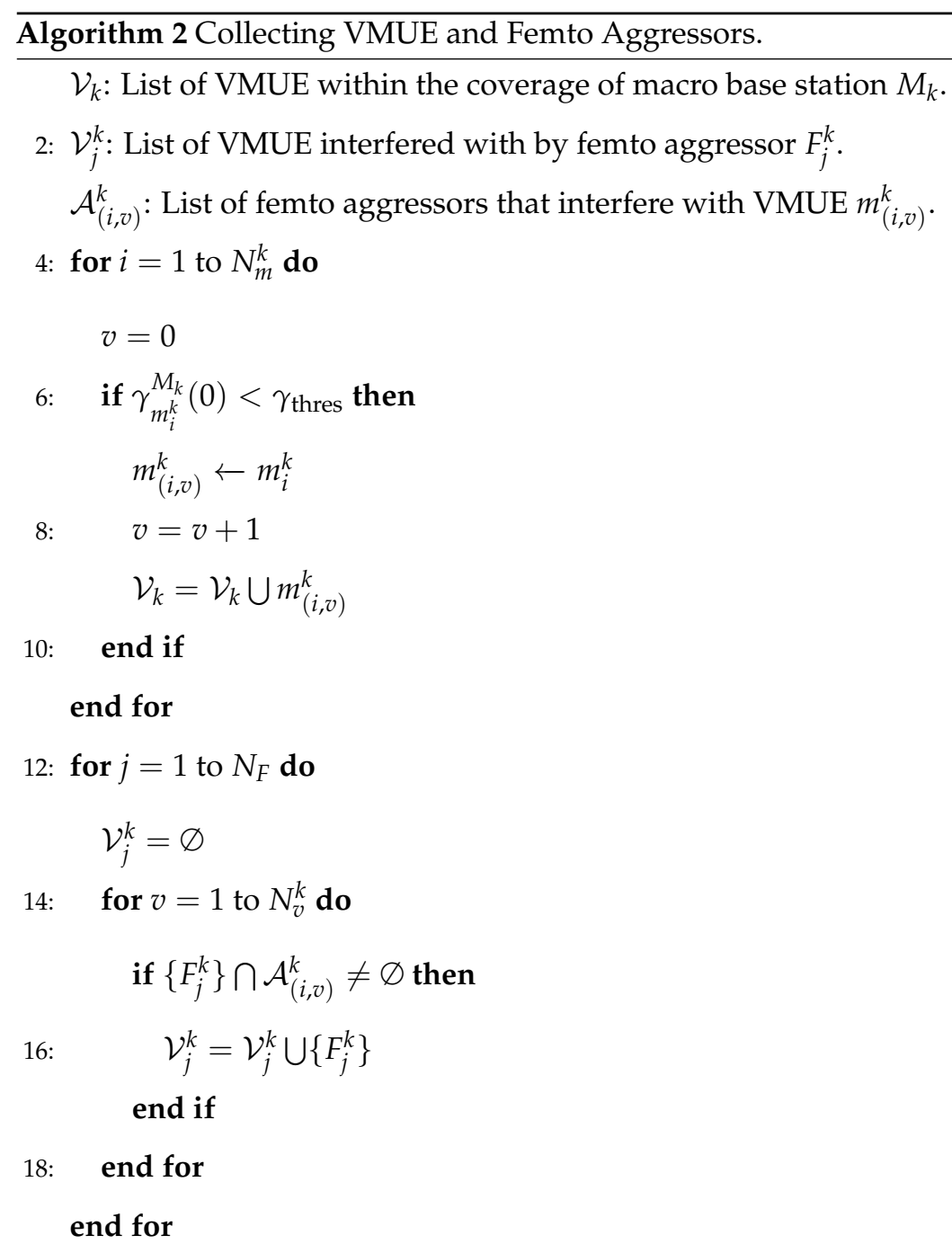






Figure 2. System model.

\subsection{Selecting Victim UE to Assist}

In an empirical network, there are numerous VMUE suffering from the interference of femto aggressors. It is not always possible to assist all VMUE, and therefore, selecting the VMUE to assist is crucial. Due to the fact that VMUE suffering from the most severe interference requires the highest ABSF ratio, as a result, a macro base station gives a higher priority to assisting VMUE with lowest the SINR values in order to reduce the required ABSF ratio. For each cluster of femto aggressors, based on the reported SINR values, a macro base station sorts all VMUE in increasing order, and assists those with the lowest SINR.

Figure 3 shows an example in which three VMUE $m_{(1,1)}^{1}, m_{(2,2)}^{1}$, and $m_{(3,3)}^{1}$ suffer from interference from a cluster including three femto aggressors $F_{1}^{1}, F_{2}^{1}$, and $F_{3}^{1}$. As can be seen from the figure, $m_{(2,2)}^{1}$ experiences the strongest interference and has the lowest SINR value. To satisfy the requirements of all three VMUE, femto base stations should be muted with an ABSF ratio that is high enough to satisfy $m_{(2,2)}^{1}$. Therefore, it is recommended to give higher priority to assisting $m_{(2,2)}^{1}$ but not the two other pieces of VMUE to effectively reduce the required ABSF ratio.

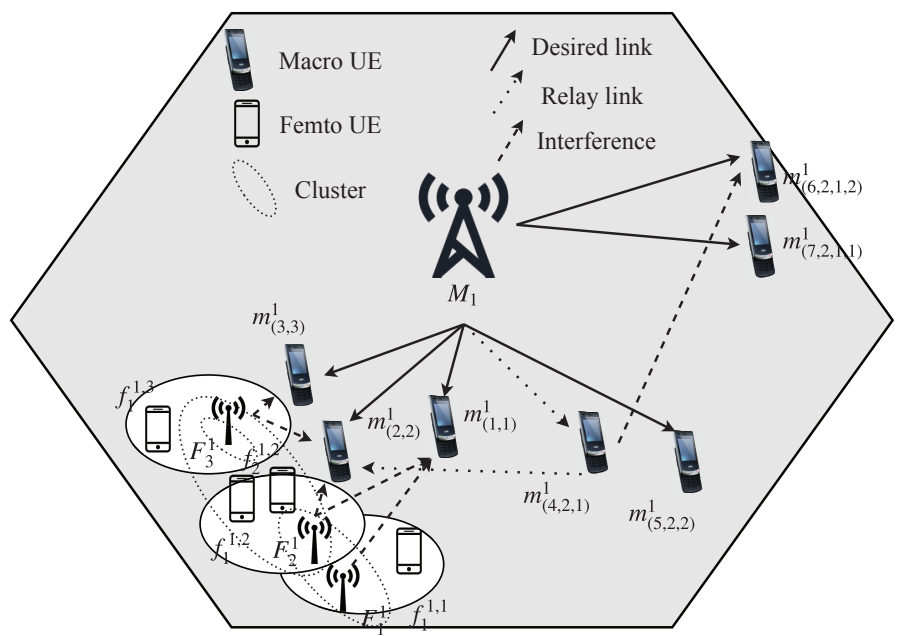

Figure 3. Example of selecting victim user equipment (UE) to assist. 


\subsection{Relay Selection}

After choosing the VMUE to assist, appropriate relay nodes for this VMUE should be considered, taking into account the interference constraints. Let $d$ denote the distance between a relay node and the assisted VMUE. In Reference [30], it was suggested that $d \leq 25 \mathrm{~m}$. The lower the value $d$, the lower the transmit power required to achieve a certain level of SINR value of VMUE, resulting in less interference (caused by the relay function) with other macro UE. Conversely, a longer distance $d$ demands higher transmit power, which consumes more power in the relay node.

In Figure 3, to assist VMUE $m_{(2,2)}^{1}$, the macro base station may select one of two relay nodes $m_{(4,2,1)}^{1}$ or $m_{(5,2,2)}^{1}$. Aiming to achieve a higher SINR value of VMUE and to lower the interference with other macro UE, relay node $m_{(4,2,1)}^{1}$, with a shorter distance to VMUE $m_{(2,2)}^{1}$, is the most appropriate. The algorithm for relay selection is given in Algorithm 3.

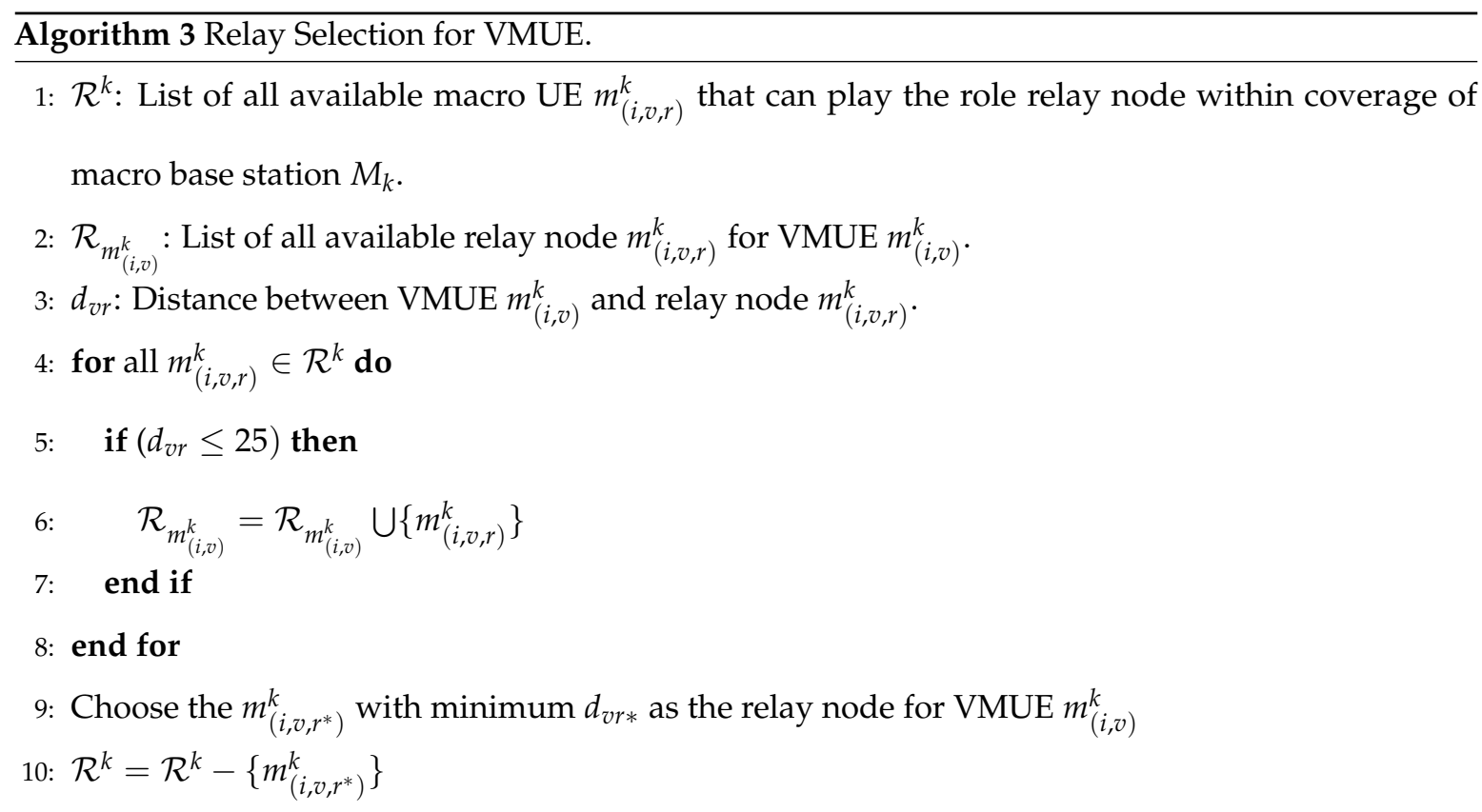

\subsection{Resource Allocation for Relay Function}

Assigning inband underlaying spectrum [21] to relay function causes interference between relay nodes and macro UE that share resource blocks with relay nodes. In this step, the most suitable resource is allocated to the relay function in such a way that the lowest amount of interference is left on other macro UE. In the literature, there are different methods to allocate resource blocks to minimize the interference caused by a relay function [31]. In this paper, we consider the mechanism in [32], called the "Greedy Heuristic resource block selection algorithm", to allocate resource blocks to the relay function. From (16) and (17), it is suggested that lower channel gain $G_{\left(m_{(i, v, r)}^{k}, m_{(i, v, r, s)}^{k}\right)}$ between relay node $m_{(i, v, r)}^{k}$ and affected macro UE $m_{(i, v, r, s)}^{k}$ results in lower interference with $m_{(i, v, r, s)}^{k}$, leading to a higher SINR value of $\gamma_{m_{(i, v, r, s)}^{k}}(0)$. Therefore, any macro UE with a higher channel quality indicator (CQI) can share its resource blocks with a relay node with a lower channel gain between them.

For example, in Figure 3, we assign the most appropriate resource block for the link from relay node $m_{(4,2,1)}^{1}$ to VMUE $m_{(2,2)}^{1}$. Macro UE $m_{(6,2,1,2)}^{1}$ and $m_{(7,2,1,1)}^{1}$ can share their resource blocks to relay node $m_{(4,2,1)}^{1}$ if their CQI values are higher than the channel gain between them and relay node $m_{(4,2,1)}^{1}$. Since $G\left(m_{(4,2,1)}^{1}, m_{(7,2,1,1)}^{1}\right)$ is greater than $G\left(m_{(4,2,1)}^{1}, m_{(6,2,1,2)}^{1}\right)$, macro UE $m_{(6,2,1,2)}^{1}$ is selected to share its resource blocks to relay node $m_{(4,2,1)}^{1}$. 


\subsection{Optimal Transmit Power}

This section explains the constraints on transmit power for the relay function. On the one hand, the higher transmit power of relay nodes $m_{(i, v, r)}^{k}$ s gives rise to a higher SINR of VMUE. On the other hand, increasing transmit power causes more severe interference with the affected UE, where resource blocks are shared to the relay function. Transmit power $P_{m_{(i, v, r)}^{k}}$ of relay node $m_{(i, v, r)}^{k}$ is decided upon to maximize the SINR of VMUE $m_{(i, v)}^{k}$ while still satisfying the SINR condition of affected macro UE $m_{(i, v, r, s)}^{k}$. From (14) and (16), these constraints are expressed as

$$
\begin{array}{ll}
\max & \gamma_{m_{(i, v)}^{k}}^{m_{i, r}^{k}}(0) \\
\text { s.t. } & \gamma_{m_{(i, v, r, s)}^{k}}^{k}(0) \geq \gamma_{\text {thres }}, \\
& P_{m_{(i, v, r)}^{k} \leq P_{\max },}
\end{array}
$$

where $P_{\max }$ is the maximum power that a relay node is capable of transmitting. The problem can be expressed in a matrix form as

$$
\begin{array}{ll}
\min & -A^{T} P_{m_{(i, v, r)}^{k}} \\
\text { s.t. } & B^{T} P_{m_{(i, v, r)}^{k}}+C+D \leq 0, \\
& P_{m_{(i, v, r)}^{k}} \leq P_{\max },
\end{array}
$$

where

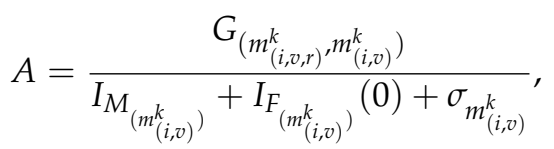

$$
\begin{aligned}
& B=G_{\left.\left(m_{(i, v, r}^{k}\right)^{k} m_{(i, v, r, s)}^{k}\right)^{\prime}} \\
& C=I_{M_{\left(m_{(i, v, r, s)}^{k}\right)}}+I_{F_{\left(m_{(i, v, r, s)}^{k}\right)}}(0)+\sigma_{\left.m_{(i, v, r, s)}^{k}\right)^{\prime}} \\
& D=-\frac{P_{M_{k}} G_{\left(M_{k}, m_{(i, v, r, s)}^{k}\right)} .}{\gamma_{\text {thres }}} .
\end{aligned}
$$

The optimal transmit power is

$$
P_{m_{(i, v, r)}^{k}}^{*}=\min \left(\frac{-(C+D)}{B}, P_{\max }\right)
$$

The resource allocation and adjustment of transmit power for the relay function is given in Algorithm 4.

\subsection{Deciding to Enable Relay or Not}

After determining the appropriate transmit power, we estimate the SINR value of VMUE if they are served by relay nodes. At this point, it is necessary to decide whether to enable the relay function to assist VMUE or not. The relay function should be enabled if

$$
\gamma_{m_{(i, v)}^{k}}^{m_{(i, v) r}^{k}}(0)>\gamma_{m_{(i, v)}^{k}}^{M_{k}}(0)
$$

In other words, the relay function should be used if relay nodes can serve VMUE better than a macro base station, and if other conditions are satisfied. Otherwise, the VMUE continue to associate with a macro base station. 


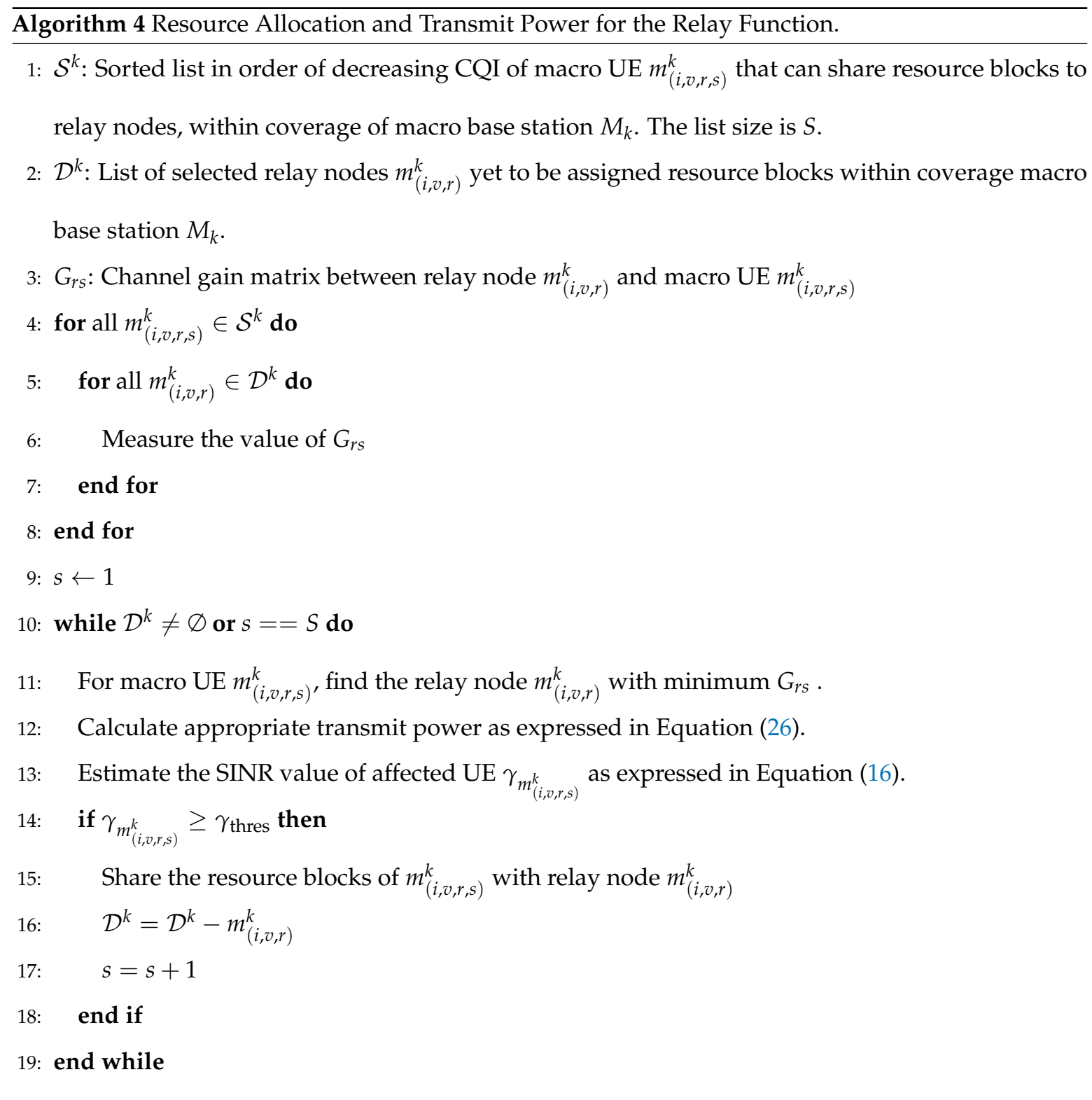

After assisting as many VMUE as possible from the victim lists, macro base stations update the list of VMUE and their status. Based on this new list, new ABSF ratios are re-calculated and applied to femto base stations. These new ABSF ratios tend to be smaller than the previous ones because the amount of VMUE has decreased, and their SINR values have improved thanks to the relay function.

\section{Simulation Results and Discussion}

In this section, we provide numerical results to evaluate the performance of the proposed algorithm. Using Matlab, we simulate and demonstrate the efficiency of the algorithm in four scenarios, with increasing complexity. Each scenario is described in one sub-section. The first scenario consists of one macro base station and one femto base station. In the second, two clusters of femto base stations are organized. Third, the algorithm is executed with 64 randomly distributed femto base stations. Lastly, we consider a case of multiple macro base stations. In each scenario, two other approaches (Non-ABSF and ABSF) are compared with the proposed ABSF and relay. To put it simply, in Non-ABSF, the network operates in a conventional fashion without any technique to guarantee the performance of the VMUE. After that, adaptive ABSF is applied to cope with intercell interference. Finally, ABSF with a 
relay function is enabled in a joint manner to assist VMUE. For performance comparisons, the following metrics were considered: user throughput, the number of QoS-satisfied-UE, and the required ABSF ratio. There are two important parameters that may leave significant impacts on performance of the proposed algorithm. The first critical parameter is the satisfied SINR threshold for a UE. Higher SINR threshold provides better quality of service to users, but also results in higher required ABSF ratio and higher transmit power of a relay function. The requirement for the SINR threshold is either -6 or $-4 \mathrm{~dB}$ according to the Third Generation Partnership Project standardization [33]. In our simulation, the value of the SINR threshold is set equal to $-4 \mathrm{~dB}$. The second parameter worth mentioning is the maximum distance between a relay node and a VMUE. Higher maximum distance increases the probability of successfully finding a relay node but also leads to higher interference and vice versa [30].

\subsection{Single Femto Base Station}

In the first scenario, an operation with one macro base station and one femto base station was simulated. Figure 4 shows the throughput of macro UE, femto UE as well as total network throughput. With Non-ABSF, the throughput of macro UE was limited because of interference, and was much lower than that of femto UE. When adaptive ABSF was activated, the throughput of macro UE recovered, but ABSF also degraded the performance of the femto base stations, leading to a remarkable decrease in the throughput of femto UE. After that, with the assistance of a relay function, the VMUE meet the SINR condition with a lower required ABSF ratio, resulting in higher throughput for femto UE in comparison with ABSF alone.

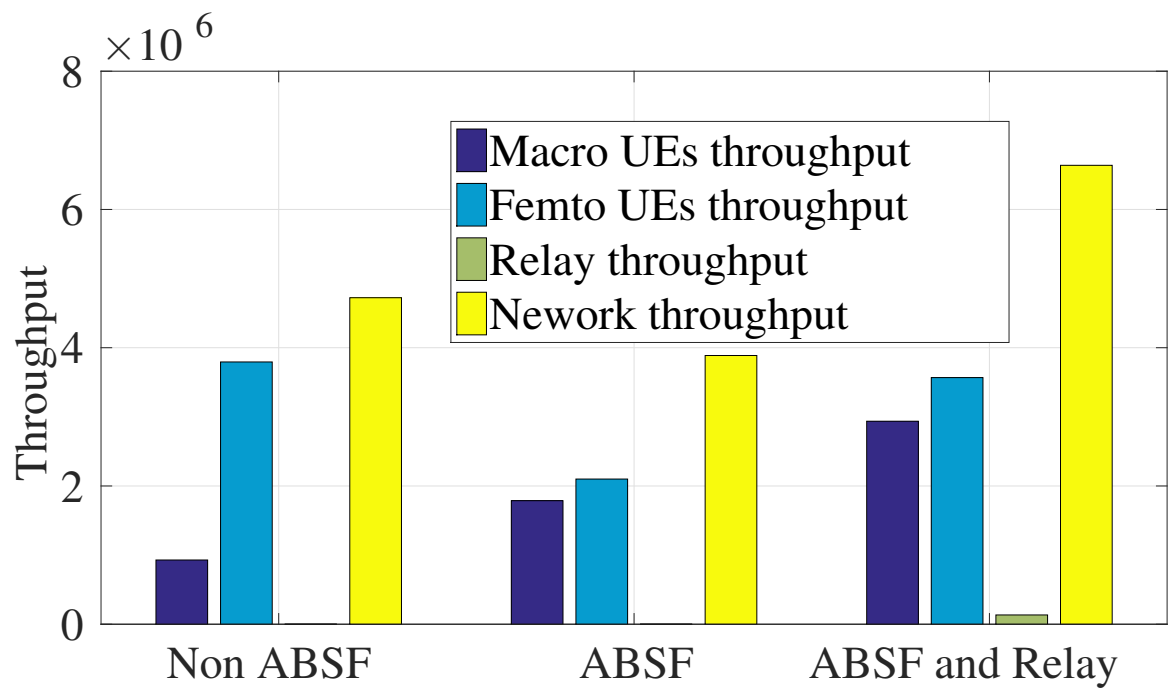

Figure 4. Single femto base station: network throughput.

Figure 5 depicts the number of QoS-satisfied-UE, of which the SINR values were higher than the SINR threshold $\gamma_{\text {thres }}$. Without ABSF, interference from femto aggressors limited the number of QoS-satisfied-macro-UE. ABSF helped to improve the performance of VMUE by muting some subframes of femto aggressors, resulting in a greater number of QoS-satisfied-macro-UE. However, blank subframes of femto base stations lowered the number of QoS-satisfied-femto-UE. By jointly considering ABSF and a relay function, the network can satisfy the requirements of VMUE without overly limiting the performance of femto UE. In Figure 6, a reduction of the required ABSF ratio is observed between ABSF and ABSF plus relay. This decrease explains the improvements in terms of throughput and the number of QoS-satisfied-UE, as shown in previous results. 


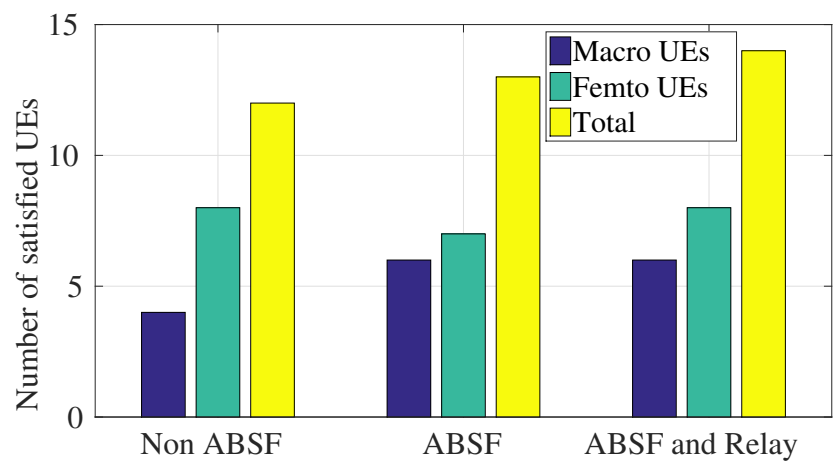

Figure 5. Single femto base station: number of QoS-satisfied-UEs.

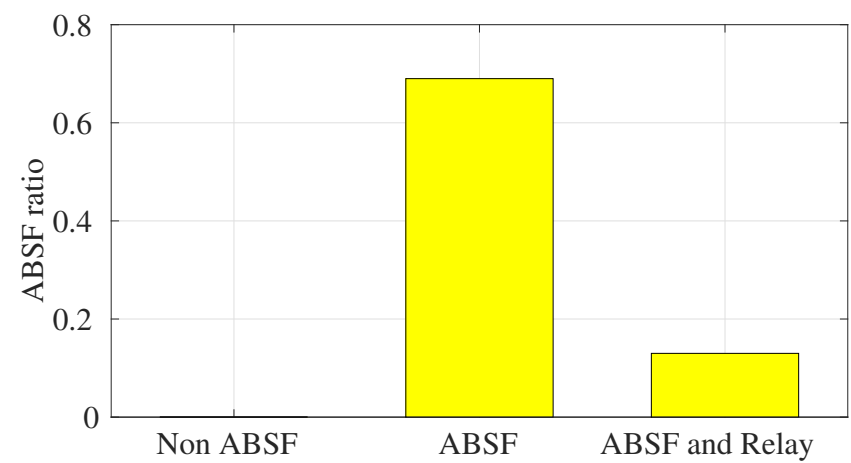

Figure 6. Single femto base station: required almost blank subframe (ABSF) ratio.

\subsection{Clusters of Femto Base Stations}

We evaluated the performance of the proposed algorithm in a more complicated scenario, consisting of five femto base stations divided into two clusters of two and three femto base stations. In an empirical network, a critical VMUE suffers from interference from a cluster of multiple femto aggressors. The ABSF ratio was determined and applied to all femto aggressors in the cluster. In other words, to satisfy a critical VMUE, depending on its channel quality and interference level, the macro base station may have to sacrifice the capacity of many femto users served by those femto aggressors. Therefore, assistance from a relay function is especially useful in situations where critical VMUE are interfered with by multiple femto aggressors. This advantage is demonstrated not only by the increase in the network throughput and the number of satisfied UEs, illustrated in Figures 7 and 8, but also by the decrease in the average required ratio, as shown in Figure 9.

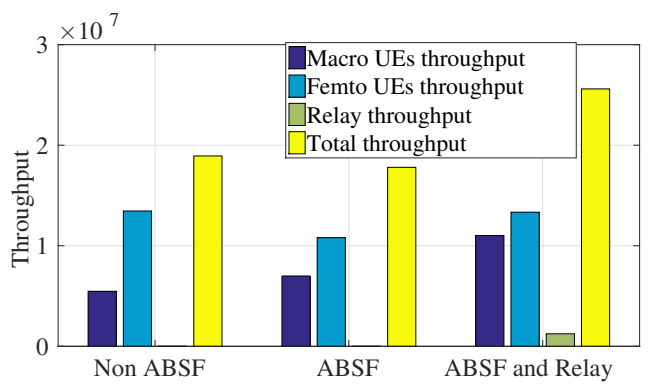

Figure 7. Clusters of femto base stations: network throughput.

\subsection{Randomly Distributed Environment}

We demonstrate the efficiency of the proposed algorithm on a network with a single macro base station and randomly distributed femto base stations. In Figures 10 and 11, with a large number of 
femto base stations and UE, the proposed algorithm exhibited increases of $37 \%$ and $4 \%$ in network throughput and the quantity of QoS-satisfied-UE, respectively, in comparison with the conventional ABSF. In fact, assisting VMUE with a relay function is not always successful because of difficulties in seeking potential relay nodes, in resource allocation, or in deciding on transmit power. Nonetheless, a substantial reduction, from 0.4 to 0.05 , in the average ABSF ratio of 64 femto base stations was achieved, as shown in Figure 12.

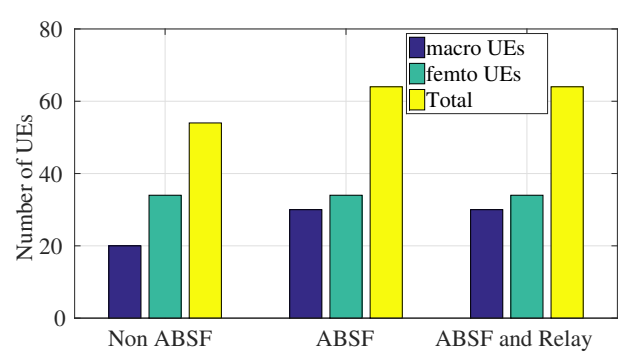

Figure 8. Clusters of femto base stations: number of QoS-satisfied-UE.

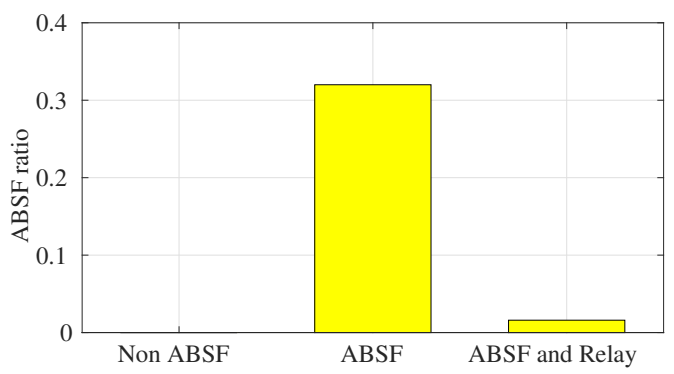

Figure 9. Clusters of femto base stations: average ABSF ratio.

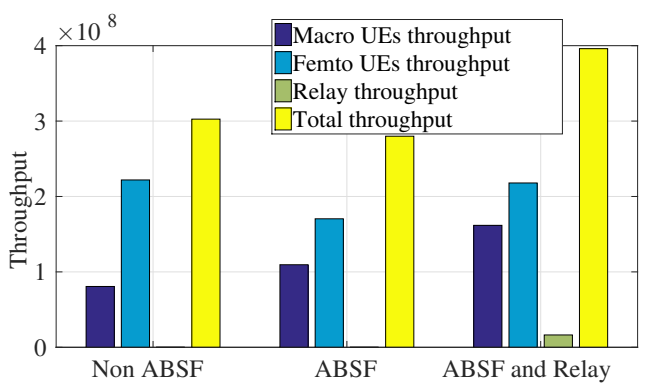

Figure 10. Sixty-four femto base stations: network throughput.



Figure 11. Sixty-four femto base stations: number of QoS-satisfied-UE. 


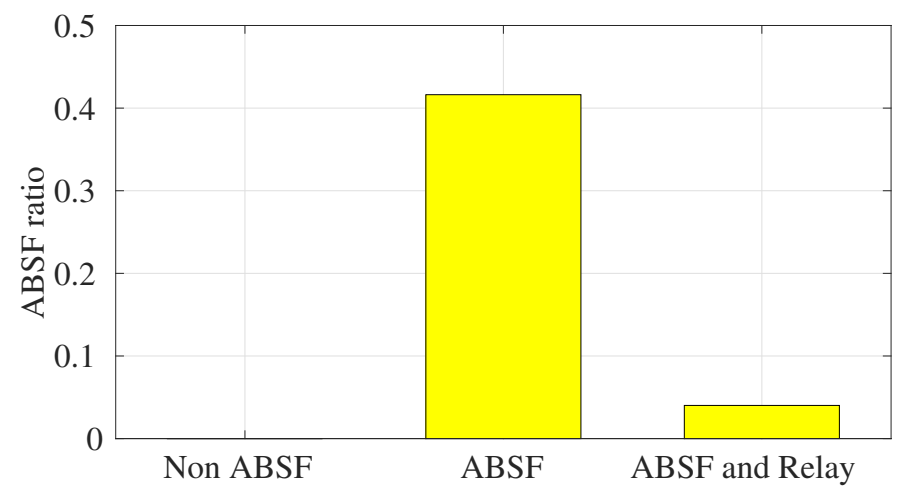

Figure 12. Sixty-four femto base stations: average ABSF ratio.

\subsection{Randomly Distributed Environment with Multiple Macro Base Stations}

Finally, we considered a general case with mutiple macrocells. In each macrocell, there were 32 randomly distributed femto base stations. Figures 13 and 14 show increases of $18 \%$ and $8 \%$ in terms of total network throughput and the number of satisfied users, respectively. In Figure 15, due to higher interference from neighboring macro base stations, VMUE experienced a lower SINR level, and hence, required a higher ABSF ratio. Thanks to the assistance of the relay function, macro base stations reduced the required ABSF ratio, providing better throughput and service quality.

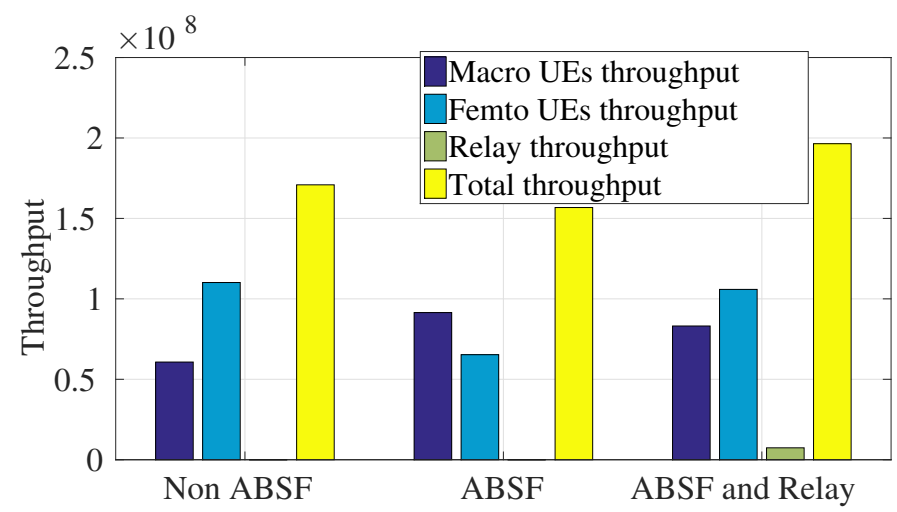

Figure 13. Multiple macro base stations, 32 femto base stations per macro base station: network throughput.

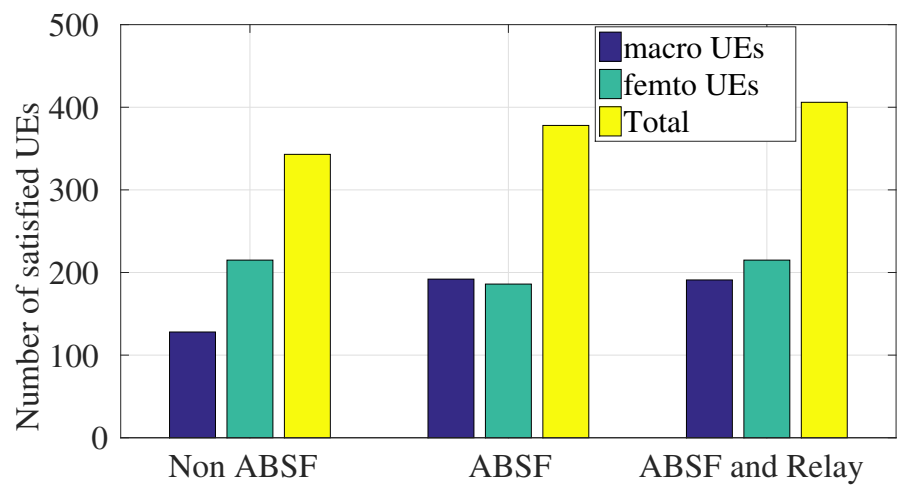

Figure 14. Multiple macro base stations, 32 femto base stations per macro base station: QoS-satisfied-UE. 


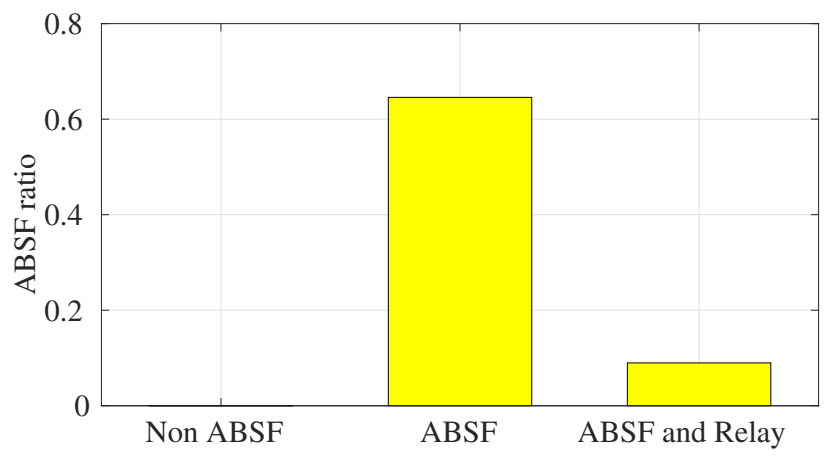

Figure 15. Multiple macro base stations, 32 femto base stations per macro base station: average ABSF ratio.

\section{Conclusions}

In this paper, we proposed an algorithm to jointly deploy an ABSF and a relay function in an LTE HetNet to provide satisfactory performance in VMUE while reducing the capacity degradation of femto UE that is caused by the ABSF. By assisting VMUE with a relay function, the required ABSF ratio to mitigate interference is lowered. In particular, starting by selecting the critical victim users to assist, the relay is then selected. After that, by considering resource allocation and power control for the relay function, the minimum QoS of victim users is guaranteed, while the interference of the relay function affecting other macro users is minimized. The simulation results show that the proposed scheme increases network throughput and the quantity of QoS-satisfied-UE by $18 \%$ and $8 \%$, respectively, compared to conventional ABSF.

Author Contributions: Conceiving the method and writing the manuscript: L.N.; results analysis: L.N. and S.K.; writing-review and editing: L.N. and S.K.; supervision: S.K. All authors have read and agreed to the published version of the manuscript.

Funding: This work has been supported by the 2020 Research Fund of the University of Ulsan.

Conflicts of Interest: The authors declare that they have no competing interests.

\section{Abbreviations}

The following abbreviations are used in this manuscript:

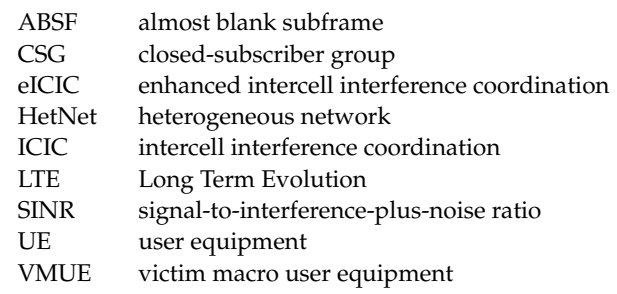

\section{References}

1. Cisco, C. Cisco Visual Networking Index: Global Mobile Data Traffic Forecast Update, 2016-2021 White Paper; Cisco: San Jose, CA, USA, 2017.

2. Li, Q.C.; Niu, H.; Papathanassiou, A.T.; Wu, G. 5G network capacity: Key elements and technologies. IEEE Veh. Technol. Mag. 2014, 9, 71-78. [CrossRef]

3. Lopez-Perez, D.; Guvenc, I.; De la Roche, G.; Kountouris, M.; Quek, T.Q.; Zhang, J. Enhanced intercell interference coordination challenges in heterogeneous networks. IEEE Wirel. Commun. 2011, 18, 22-30. [CrossRef]

4. Damnjanovic, A.; Montojo, J.; Wei, Y.; Ji, T.; Luo, T.; Vajapeyam, M.; Yoo, T.; Song, O.; Malladi, D. A survey on 3GPP heterogeneous networks. IEEE Wirel. Commun. 2011, 18, 10-21. [CrossRef] 
5. Bhushan, N.; Li, J.; Malladi, D.; Gilmore, R.; Brenner, D.; Damnjanovic, A.; Sukhavasi, R.T.; Patel, C.; Geirhofer, S. Network densification: The dominant theme for wireless evolution into 5G. IEEE Commun. Mag. 2014, 52, 82-89. [CrossRef]

6. Andrews, J.G.; Claussen, H.; Dohler, M.; Rangan, S.; Reed, M.C. Femtocells: Past, present, and future. IEEE J. Sel. Areas Commun. 2012, 30, 497-508. [CrossRef]

7. Hasan, M.M.; Kwon, S.; Oh, S. Frequent-handover mitigation in ultra-dense heterogeneous networks. IEEE Trans. Veh. Technol. 2018, 68, 1035-1040. [CrossRef]

8. Duong, T.M.; Kwon, S. Vertical handover analysis for randomly deployed small cells in heterogeneous networks. IEEE Trans. Wirel. Commun. 2020, 19, 2282-2292. [CrossRef]

9. Hasan, M.M.; Kwon, S.; Na, J.H. Adaptive mobility load balancing algorithm for LTE small-cell networks. IEEE Trans. Wirel. Commun. 2018, 17, 2205-2217. [CrossRef]

10. Saquib, N.; Hossain, E.; Kim, D.I. Fractional frequency reuse for interference management in LTE-advanced hetnets. IEEE Wirel. Commun. 2013, 20, 113-122. [CrossRef]

11. Khandekar, A.; Bhushan, N.; Tingfang, J.; Vanghi, V. LTE-advanced: Heterogeneous networks. In Proceedings of the 2010 European Wireless Conference (EW), Lucca, Italy, 12-15 April 2010; IEEE: Piscataway, NI, USA, 2010; pp. 978-982.

12. Barbieri, A.; Damnjanovic, A.; Ji, T.; Montojo, J.; Wei, Y.; Malladi, D.; Song, O.; Horn, G. LTE femtocells: System design and performance analysis. IEEE J. Sel. Areas Commun. 2012, 30, 586-594. [CrossRef]

13. Hamza, A.S.; Khalifa, S.S.; Hamza, H.S.; Elsayed, K. A survey on inter-cell interference coordination techniques in OFDMA-based cellular networks. IEEE Commun. Surv. Tutorials 2013, 15, 1642-1670. [CrossRef]

14. Deb, S.; Monogioudis, P.; Miernik, J.; Seymour, J.P. Algorithms for enhanced inter-cell interference coordination (eICIC) in LTE HetNets. IEEE/ACM Trans. Netw. 2014, 22, 137-150. [CrossRef]

15. Kamel, M.I.; Elsayed, K.M. Performance evaluation of a coordinated time-domain eICIC framework based on ABSF in heterogeneous LTE-advanced networks. In Proceedings of the 2012 IEEE Global Communications Conference (GLOBECOM), Anaheim, CA, USA, 3-7 December 2012; pp. 5326-5331.

16. Zhang, J.; Tian, H.; Tian, P.; Huang, Y.; Gao, L. Dynamic frequency reservation scheme for interference coordination in LTE-advanced heterogeneous networks. In Proceedings of the 2012 IEEE 75th Vehicular Technology Conference (VTC Spring), Yokohama, Japan, 6-9 May 2012; IEEE: Piscataway, NJ, USA, 2012; pp. 1-5.

17. Lembo, S.; Lunden, P.; Tirkkonen, O.; Valkealahti, K. Optimal muting ratio for enhanced inter-cell interference coordination (eICIC) in HetNets. In Proceedings of the IEE ICC, Budapest, Hungary, 9-13 June 2013; pp. 1145-1149.

18. Cierny, M.; Wang, H.; Wichman, R.; Ding, Z.; Wijting, C. On number of almost blank subframes in heterogeneous cellular networks. IEEE Trans. Wirel. Commun. 2013, 12, 5061-5073. [CrossRef]

19. Merwaday, A.; Mukherjee, S.; Güvenç, I. Capacity analysis of LTE-Advanced HetNets with reduced power subframes and range expansion. Eurasip J. Wirel. Commun. Netw. 2014, 2014, 189. [CrossRef]

20. Nguyen, L.H.; Nguyen, M.T.; Kwon, S. Adaptive Reduced Power Subframe in LTE Heterogeneous Networks. In Proceedings of the Fall Conference Proc. of Korea Communications Society; 2018; pp. 73-74. Available online: http:/ / www.dbpia.co.kr/Journal/articleDetail?nodeId=NODE07564999 (accessed on 18 August 2020).

21. Mach, P.; Becvar, Z.; Vanek, T. In-band device-to-device communication in OFDMA cellular networks: A survey and challenges. IEEE Commun. Surv. Tutor. 2015, 17, 1885-1922. [CrossRef]

22. Liu, J.; Zhang, S.; Kato, N.; Ujikawa, H.; Suzuki, K. Device-to-device communications for enhancing quality of experience in software defined multi-tier LTE-A networks. IEEE Netw. 2015, 29, 46-52. [CrossRef]

23. Liu, J.; Kawamoto, Y.; Nishiyama, H.; Kato, N.; Kadowaki, N. Device-to-device communications achieve efficient load balancing in LTE-advanced networks. IEEE Wirel. Commun. 2014, 21, 57-65. [CrossRef]

24. Choi, S.K.; Kim, W.J.; Lee, H.S.; Kim, D.I. Interference forwarding for D2D based heterogeneous cellular networks. In Proceedings of the 2013 IEEE/CIC International Conference on Communications in China (ICCC), Xi'an, China, 12-14 August 2013; pp. 130-134.

25. Dang, H.P.; Van Nguyen, M.S.; Do, D.T.; Pham, H.L.; Selim, B.; Kaddoum, G. Joint Relay Selection, Full-Duplex and Device-to-Device Transmission in Wireless Powered NOMA Networks. IEEE Access 2020, 8, 82442-82460. [CrossRef]

26. Yeh, S.P.; Talwar, S.; Wu, G.; Himayat, N.; Johnsson, K. Capacity and coverage enhancement in heterogeneous networks. IEEE Wirel. Commun. 2011, 18, 32-38. [CrossRef] 
27. Vu, T.K.; Kwon, S.; Oh, S. Cooperative Interference Mitigation Algorithm in Heterogeneous Networks. IEICE Trans. Commun. 2015, 98, 2238-2247. [CrossRef]

28. Jang, J.; Lee, K.B. Transmit power adaptation for multiuser OFDM systems. IEEE J. Sel. Areas Commun. 2003, 21, 171-178. [CrossRef]

29. Kwon, S.; Shroff, N.B. Energy-efficient interference-based routing for multi-hop wireless networks. In Proceedings of the INFOCOM, Barcelona, Spain, 23-29 April 2006; pp. 1-12.

30. Doppler, K.; Rinne, M.; Wijting, C.; Ribeiro, C.B.; Hugl, K. Device-to-device communication as an underlay to LTE-advanced networks. IEEE Commun. Mag. 2009, 47, 42-49. [CrossRef]

31. Asadi, A.; Wang, Q.; Mancuso, V. A survey on device-to-device communication in cellular networks. IEEE Commun. Surv. Tutor. 2014, 16, 1801-1819. [CrossRef]

32. Zulhasnine, M.; Huang, C.; Srinivasan, A. Efficient resource allocation for device-to-device communication underlaying LTE network. In Proceedings of the 2010 IEEE 6th International Conference on Wireless and Mobile Computing, Networking and Communications, Chengdu, China, 23-25 September 2010; pp. 368-375.

33. Yang, T.; Zhang, L. Approaches to enhancing autonomous power control at femto under co-channel deployment of macrocell and femtocell. In Proceedings of the 2011 IEEE 22nd International Symposium on Personal, Indoor and Mobile Radio Communications, Toronto, ON, Canada, 11-14 September 2011; IEEE: Piscataway, NJ, USA, 2011; pp. 71-75.

(c) 2020 by the authors. Licensee MDPI, Basel, Switzerland. This article is an open access article distributed under the terms and conditions of the Creative Commons Attribution (CC BY) license (http:// creativecommons.org/licenses/by/4.0/). 